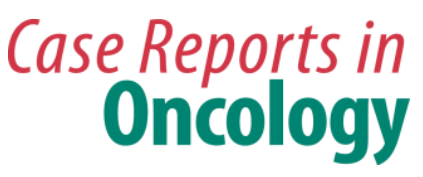

Case Rep Oncol 2016;9:854-860

DOI: $10.1159 / 000453255$

Publisnea oninne: December 14, 2016
(C) 2016 The Author(s)

Published by S. Karger AG, Basel www.karger.com/cro

This article is licensed under the Creative Commons Attribution-NonCommercial 4.0 International License (CC BY-NC) (http://www.karger.com/Services/OpenAccessLicense). Usage and distribution for commercial purposes requires written permission.

\title{
A Patient with Supraclavicular Lymphadenopathy and Anterior Mediastinal Mass Presenting as a Rare Case of Composite Lymphoma: A Case Report and Literature Review
}

\author{
Alex Raufi $^{a} \quad$ James Jerkins $^{b} \quad$ Yung Lyou $^{b} \quad$ Deepa Jeyakumar $^{b}$ \\ ${ }^{a}$ Department of Medicine, University of California Irvine Medical Center, Orange, CA, USA; \\ ${ }^{b}$ Department of Medicine, Division of Hematology and Oncology, University of California \\ Irvine Medical Center, Orange, CA, USA
}

\section{Keywords}

Composite lymphoma $\cdot$ Supraclavicular lymphadenopathy $\cdot$ Anterior mediastinal mass

\begin{abstract}
Composite lymphoma $(\mathrm{CL})$ is a rare disease with 2 distinct lymphomas concurrently arising in a single patient with an estimated incidence of $1-4.7 \%$ of newly diagnosed lymphomas per year. $\mathrm{CL}$ most commonly involves $2 \mathrm{~B}$-cell non-Hodgkin lymphomas $(\mathrm{NHL})$ or a $\mathrm{B}$-cell $\mathrm{NHL}$ with a Hodgkin lymphoma. Our case is unique in that it was a bilineage $\mathrm{CL}$ with both a $\mathrm{T}$-cell and B-cell NHL, which has only been reported in a few case reports. A 49-year-old woman presented with several months of progressive cough, weight loss, dyspnea, and supraclavicular lymphadenopathy. Computed tomographic imaging done upon admission to the hospital found that she had extensive anterior and middle mediastinal lymphadenopathy as well as bilateral supraclavicular lymphadenopathy. The patient underwent an excisional biopsy on the supraclavicular lymph node and was found to have a composite lymphoma involving both a T-cell and B-cell NHL. Her final pathological diagnosis was peripheral T-cell lymphoma and lymphoplasmacytic lymphoma. The patient was found to have stage IIIB disease. Her
\end{abstract}


Raufi et al:: A Patient with Supraclavicular Lymphadenopathy and Anterior Mediastinal Mass Presenting as a Rare Case of Composite Lymphoma

HIV, hepatitis panel, and tuberculosis tests were all negative. She then underwent chemotherapy with dose-adjusted EPOCH-R (etoposide, prednisone, vincristine, cyclophosphamide, doxorubicin, and rituximab). The patient showed a complete response and was then referred to a bone marrow transplant center for an autologous hematopoietic stem cell transplant. CL is a rare disease composed of at least 2 distinct lymphomas concurrently arising in a single patient. Due to the complexity in having to treat multiple types of lymphoma simultaneously $\mathrm{CL}$ presents challenges with treatment and assessing prognosis.

(C) 2016 The Author(s)

Published by S. Karger AG, Basel

\section{Introduction}

The term "composite lymphoma" (CL) was introduced by Custer [1] in the mid-1950s to describe cases of 2 distinct lymphomas concurrently arising in a single patient. The incidence of CL is low with an estimated 1-4.7\% of lymphoma cases representing CL [2]. Typically, CL consists of 2 distinct non-Hodgkin lymphomas (NHL) or a classic Hodgkin lymphoma with an NHL; however, case reports of 2 Hodgkin lymphomas also exist. In cases of lymphoma, diagnostic accuracy relies heavily on a pathologist's expertise and is of great clinical consequence as it determines prognosis and selection of treatment regimen. CL presents a unique challenge to clinicians as little data exist in regards to treatment protocols and outcomes. Here we discuss a case of a CL involving both a T-cell and a B-cell NHL and we will review the literature on the diagnostic evaluation and treatment of such a CL.

\section{Case Report}

A 49-year-old woman recently diagnosed with anemia presented to the University of California Irvine Medical Center with a chief complaint of progressively worsening cough. She described her cough, which began a month prior to her admission, as initially mild and dry; however, 2 days prior to admission, her symptoms changed and her cough became both more intense and productive of white sputum. On the day of admission, she began experiencing shortness of breath associated with subjective fevers and chills, prompting her to seek medical attention. Upon further questioning, she disclosed decreased appetite and approximately 13 pounds of weight loss over the last month. She denied night sweats, sick contacts, or prior tuberculosis exposure. Her most recent travel outside of the US was to Vietnam 5 years prior. Anemia had been diagnosed 1 month ago by her primary care physician; at that time, her hemoglobin level was $9 \mathrm{mg} / \mathrm{dL}$ and this was attributed to heavy menses. The patient also denied a history of smoking, radiation exposure, or a personal or family history of malignancy.

On admission, the patient was tachycardic and febrile. She had dry mucous membranes and a large palpable fixed cervical lymph node. No hepatosplenomegaly was found. Initial blood work revealed a hemoglobin level of $6 \mathrm{mg} / \mathrm{dL}$ with an MCV of $71.0 \mathrm{fL}$, but no leukocytosis. There was moderate anisocytosis, slight polychromasia, multiple microcytes, and rouleaux formation on peripheral blood smear. Her chemistries were notable for hyponatremia $(129 \mathrm{mEq} / \mathrm{L})$, and she had a creatinine level of $1.1 \mathrm{mg} / \mathrm{dL}$, total bilirubin of $2.6 \mathrm{mg} / \mathrm{dL}$, and LDH of $179 \mathrm{IU} / \mathrm{L}$. Liver function tests were within normal limits. Albumin level was 1.74 $\mathrm{g} / \mathrm{dL}$. Further chemistries revealed a total protein level of $10.0 \mathrm{~g} / \mathrm{dL}$ and notably elevated 


\section{Case Reports in Oncology}

Raufi et al:: A Patient with Supraclavicular Lymphadenopathy and Anterior Mediastinal Mass Presenting as a Rare Case of Composite Lymphoma

kappa (61 mg/L) levels but normal lambda (12.80 mg/L) levels. Chest radiograph showed a soft tissue density in the left hilum concerning for lymphadenopathy (Fig. 1a, b).

The patient was started on empiric antibiotics and a work-up for tuberculosis was begun. Given the significantly elevated protein levels on her serum protein electrophoresis, urine protein electrophoresis, and immunofixation were ordered and the hematology service was consulted. Serum protein electrophoresis revealed a band with restricted mobility in the gamma region as well as a band in the beta region. Urine protein electrophoresis also showed 2 bands, both with restricted mobility in the gamma region. Serum immunofixation revealed 2 IgA kappa proteins and 1 free kappa light chain monoclonal protein. Urine immunofixation showed 1 IgA kappa protein and 1 free kappa light chain monoclonal protein. A chest computed tomography (CT) to further characterize chest radiographic findings demonstrated extensive anterior and middle mediastinal lymphadenopathy as well as bilateral supraclavicular lymphadenopathy. In addition, mildly prominent left hilar and right axillary lymph nodes as well as bilateral pleural effusions were seen. Abdominal CT showed marked hepatomegaly and pleural effusions. Given these findings, an excisional biopsy on the supraclavicular lymph node was performed.

On macroscopic inspection, the biopsied lymph nodes were markedly enlarged. Microscopically, lymph node staining with hematoxylin and eosin stain revealed a sinus histiocytosis pattern where mixed B-cells were found forming aggregates and both T-cells and plasma cells were found arranged in clusters and sheets around vessels (Fig. 2). In addition, there were patchy EBV-positive cells, mostly around the follicles. T-cells formed the dominant population which did not show any aberrant loss of surface antigen expression and were positive for CD2, CD3, CD4, CD5, CD7, and CD8 both on immunohistochemistry (IHC) and flow cytometry. Samples were negative for PD1, BCL-6, and BCL-2. The B-cells were positive for CD20 and PAX-5 and some were notably enlarged and positive for ISH-EBV. CD138 and MUM-1 were used to highlight numerous plasma cells which appeared to be kappa light chain restricted. The Ki-67 level was $15-20 \%$ and stained mostly larger cells. MYD88 L265P mutation was negative, and PCR confirmed the B-cell immunoglobulin heavy change to be clonally rearranged, although $\mathrm{T}$-cell beta and gamma clonal rearrangement was not detected.

The histologic features of hypervascularity with dominant T-cell population that showed aberrant loss of BCL-2 are consistent with a T-cell lymphoma, most likely peripheral T-cell lymphoma. The presence of monotypic plasmacytic proliferation with mixed small and large B-cells is consistent with a low-grade B-cell lymphoma with exuberant plasmacytic differentiation. The differential diagnosis includes marginal zone B-cell lymphoma with increased plasma cells and lymphoplasmacytic lymphoma the latter of which was suspected in this case. Given these findings, the patient was diagnosed with CL consisting of peripheral T-cell lymphoma not otherwise specified and lymphoplasmacytic lymphoma.

The patient underwent staging bone marrow biopsy which revealed a hypercellular marrow with active trilineage hematopoiesis and moderate myeloid hyperplasia but no evidence of B-cell or T-cell lymphomatous involvement. Karyotype was 46,XX. CSF analysis also did not reveal any malignant cells by flow cytometry. CT scan of the head was within normal limits. Given these findings, the patient was deemed to have stage IIIB disease. HIV status and hepatitis panel were negative. Tuberculosis testing was negative. Dose-adjusted EPOCH$\mathrm{R}$ (etoposide, prednisone, vincristine, cyclophosphamide, doxorubicin, and rituximab) with monthly prophylactic intrathecal hydrocortisone, methotrexate, and cytarabine was administered and to date the patient has received a total of 5 cycles. PET-CT after the second cycle showed a partial response to therapy with significant reductions in both level IV node, and 
Raufi et al:: A Patient with Supraclavicular Lymphadenopathy and Anterior Mediastinal Mass Presenting as a Rare Case of Composite Lymphoma

anterior mediastinal lymph node conglomerate (Fig. 1c, d). Given the aggressive nature of her disease including the $\mathrm{T}$ cell component of her lymphoma, she was referred to a bone marrow transplant center and planned for autologous bone marrow transplant after completion of her sixth cycle of chemotherapy.

\section{Discussion}

CL is increasingly being recognized in part due to the greater use of adjunctive laboratory techniques such as flow cytometry, IHC, and molecular and cytogenetic assays. To date combinations of nearly all common lymphomas have been described in CL. By far the most commonly reported cases involve 2 B-cell NHL or a B-cell NHL with a Hodgkin lymphoma [3]. What makes this case unique is the bilineage involvement of both a T-cell NHL and a Bcell NHL. To date less than 100 such cases have been described, representing less than $1 \%$ of all reported lymphoid malignancies [3]. Among these cases, the most frequent B-cell NHL identified was diffuse large B-cell lymphoma, whereas peripheral T-cell lymphoma not otherwise specified and angioimmunoblastic T-cell lymphoma were the most commonly identified T-cell NHL [3].

The diagnosis of CL is primarily based on histology and given the complexity of biopsy tissue architecture, where mixtures of infiltrates of different lymphoma types can occur, many cases of CL are likely missed. Traditionally, hematoxylin and eosin staining had been used for the diagnosis of lymphoma. The application of IHC adds considerable diagnostic specificity being able to both confirm clonality and cell subtype. Newer molecular techniques using PCR to detect immunoglobulin heavy chain $(\operatorname{IgH})$ and T-cell receptor gene rearrangements can confirm clonalities of both B-and T-cell components and further aid in the diagnosis of a CL [4]. Flow cytometry is another ancillary method proven to have certain advantages such as the ability to distinguish between different cell phenotypes simultaneously and determine clonality of different cell populations. This was demonstrated in a study by Demurtas et al. [5] who used flow cytometry to successfully identify 17 CL cases among a total of 1,332 lymphomas. Lastly, EBV testing, as was performed in this case, has been shown to be a useful adjunct [6].

The pathogenesis of CL is poorly understood and, given its rarity, even less is known about the formation of composite T-cell and B-cell lymphomas. Although it is possible that some cases are due to mere coincidence, the majority is likely not, and multiple mechanisms have been proposed to explain how these 2 lymphomas arise simultaneously. One theory involves transformative mutations occurring in pluripotent stem cells destined to give rise to both B and T lymphocytes. This theory is supported by the discovery of syngeneic mutations present in both B-cell and T-cell populations in several CL cases. An example of this is the discovery of loss-of-function mutations in TET (ten-eleven translocation) 2, a tumor suppressor gene, which has been found to predispose both mice and humans to the development of a mixed B/T-cell CL [7]. Work by Wang et al. [3] has further supported this hypothesis with the discovery of the CCND1 gene (cyclin D1) mutations in both B- and T-cell populations. Some have suggested that chronic stimulation of B- and T-cells with either common antigens or cytokines could mediate transformation of both lineages [8]. Lastly, virally induced transformation has been suggested as a possible etiology, specifically in EBV-positive lymphomas. A study by Zettl et al. [9] which investigated 17 cases of T-cell lymphoma suggested that immunosuppression induced by a T-cell lymphoma may lead to EBV-associated B-cell lymphoma. 
Cases of CL present a unique challenge to clinicians since, aside from case reports and small case series, no large therapeutic studies have been conducted. A further complicating matter is the uncertainty that often arises when interpreting imaging studies. In cases of metastatic disease, it is often not feasible to determine which lymphoma component has spread, therefore making it difficult to stage properly. Nevertheless, the focus of therapy should be directed towards the more aggressive component of the CL. Specifically, in cases of mixed T-cell and B-cell lymphoma, some data exist to support the role of anti-CD20 antibodies in regimens; however, this again is limited to case reports [10]. Schmitz et al. [11] analyzed 320 patients with T-cell lymphomas treated within trials of the German High-Grade Non-Hodgkin Lymphoma Study Group and found a significant benefit of CHOP plus etoposide in patients aged $<60$ years with a normal LDH level.

\section{Conclusions}

As this case demonstrates, CL presents similarly to most lymphomas; however, it presents new challenges with regards to the management and prognosis. Much remains to be learned and more studies are needed to help develop treatment protocols not only for combined B-cell and T-cell CL but CL as a whole.

\section{Statement of Ethics}

The authors have no ethical conflicts to disclose. This material has not been published in whole or in part elsewhere. All authors have been personally and actively involved in substantive work leading to the manuscript and will hold themselves jointly and individually responsible for its content.

\section{Disclosure Statement}

The authors have no competing interests to report at the time of this publication.

\section{Author Contributions}

A.R. was the primary author documenting the patient's care and composing the case report. J.J. and D.J. are the attending hematologists who follow the patient regularly as an outpatient and contributed to the editing of the manuscript. Y.L. was also involved in the patient's inpatient care and editing process of the manuscript. All authors have read and approved the final version of the manuscript.

\section{References}

1 Custer R: Pitfalls in the Diagnosis of Lymphoma and Leukemia from Pathologist's Point of View. Proceedings of Second National Conference. New York, American Cancer Society, 1954, pp 554-557.

$\longrightarrow 2$ Thirumala S, Esposito M, Fuchs A: An unusual variant of composite lymphoma: a short case report and review of the literature. Arch Pathol Lab Med 2000;124:1376-1378. 


\section{Case Reports in Oncology}

\begin{tabular}{l|l}
\hline Case Rep Oncol 2016;9:854-860 \\
\hline DOI: 10.1159/000453255 & $\begin{array}{l}\text { C 2016 The Author(s). Published by S. Karger AG, Basel } \\
\text { www.karger.com/cro }\end{array}$ \\
\hline
\end{tabular}

Raufi et al.: A Patient with Supraclavicular Lymphadenopathy and Anterior Mediastinal Mass Presenting as a Rare Case of Composite Lymphoma

3 Wang E, et al: Composite lymphoid neoplasm of B-cell and T-cell origins: a pathologic study of 14 cases. Hum Pathol 2014;45:768-784.

-4 Suefuji N, et al: Clinicopathological analysis of a composite lymphoma containing both T- and B-cell lymphomas. Pathol Int 2012;62:690-698.

$\checkmark 5$ Demurtas A, et al: Usefulness of multiparametric flow cytometry in detecting composite lymphoma: study of 17 cases in a 12-year period. Am J Clin Pathol 2011;135:541-555.

-6 Papalas JA, et al: Primary cutaneous, composite, Epstein-Barr virus-associated, diffuse large B-cell lymphoma and peripheral T-cell lymphoma. Am J Dermatopathol 2011;33:719-725.

-7 Quivoron C, et al: TET2 inactivation results in pleiotropic hematopoietic abnormalities in mouse and is a recurrent event during human lymphomagenesis. Cancer Cell 2011;20:25-38.

-8 Volk AL, et al: Composite mycosis fungoides and B-cell chronic lymphocytic leukemia. Ann Diagn Pathol 2002;6:172-182.

-9 Zettl A, et al: Epstein-Barr virus-associated B-cell lymphoproliferative disorders in angloimmunoblastic T-cell lymphoma and peripheral T-cell lymphoma, unspecified. Am J Clin Pathol 2002;117:368-379.

10 Balague 0, et al: Epstein-Barr virus negative clonal plasma cell proliferations and lymphomas in peripheral T-cell lymphomas: a phenomenon with distinctive clinicopathologic features. Am J Surg Pathol 2007;31:1310-1322.

11 Schmitz N, et al: Treatment and prognosis of mature T-cell and NK-cell lymphoma: an analysis of patients with T-cell lymphoma treated in studies of the German High-Grade Non-Hodgkin Lymphoma Study Group. Blood 2010;116:3418-3425.
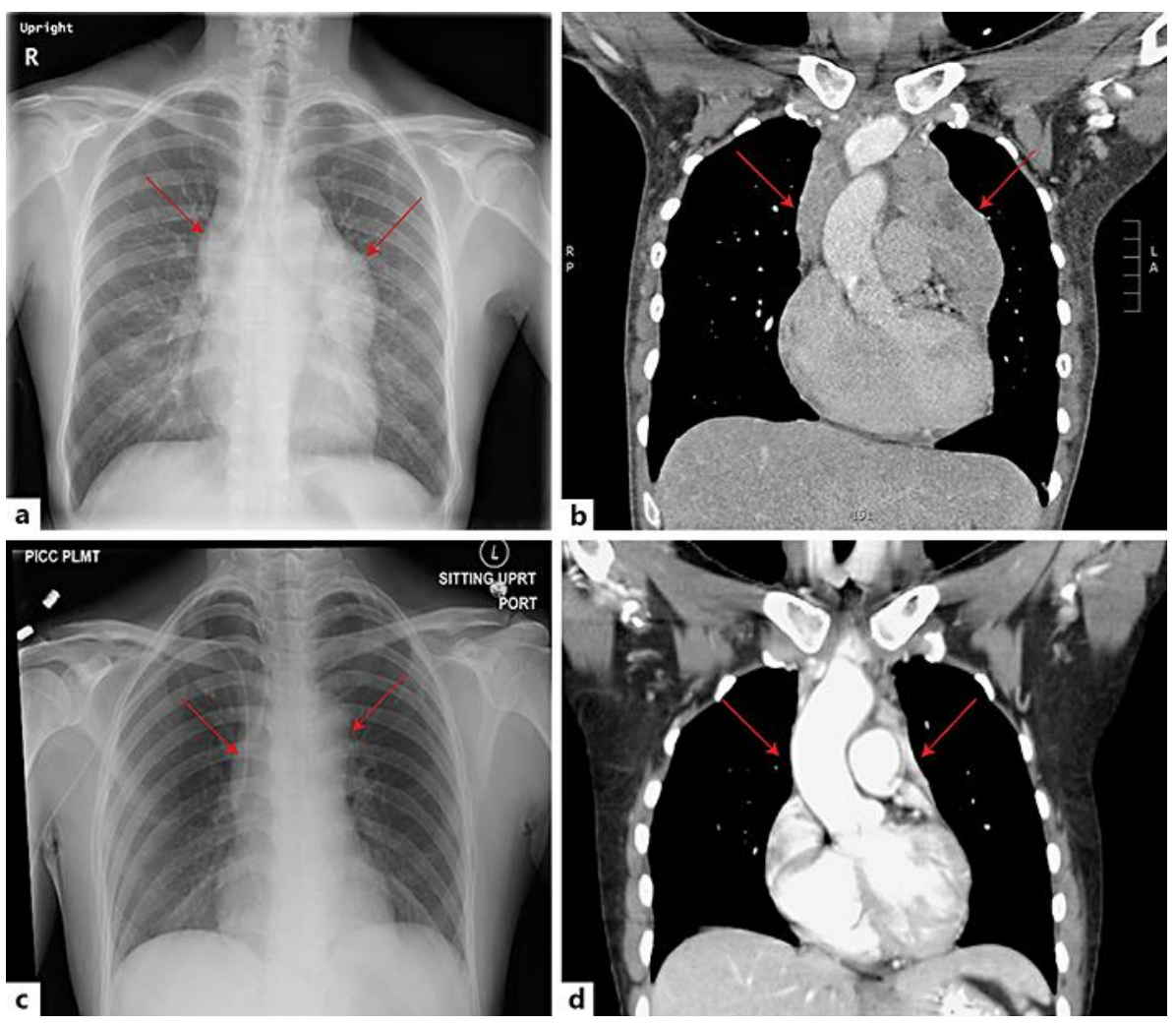

Fig. 1. Radiographic imaging before and after chemotherapy treatment. Chest X-ray (a) and CT of the chest (b) show enlarged bilateral hilar lymph nodes prior to receiving chemotherapy treatment (indicated by the red arrows). Chest X-ray (c) and CT of the chest (d) showing chemotherapy response after undergoing a full course of chemotherapy treatment. 


\section{Case Reports in Oncology}
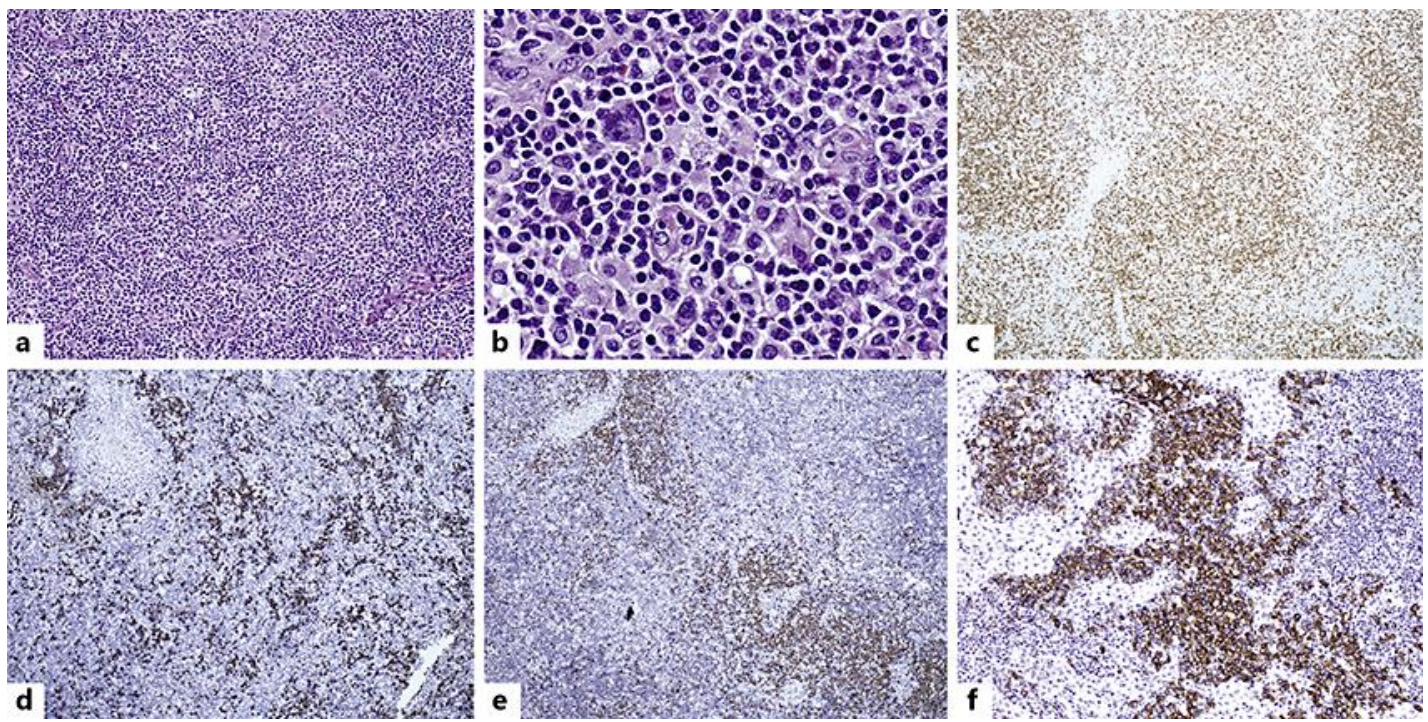

Fig. 2. Lymph node biopsy with histological staining. a, b Mixed B-cells, T-cells and plasma cells are arranged in clusters and sheets around vessels in a sinus histiocytosis pattern (hematoxylin and eosin staining, $\times 100[\mathrm{a}], \times 1,000[\mathrm{~b}])$. c T-cells positive for CD3 $(\times 100)$. d B-cells positive for CD20 $(\times 100)$. e Plasma cells positive for MUM1 $(\times 100)$. $f$ Plasma cells positive for CD138 $(\times 100)$. 